\title{
EL PAPEL DE LOS PROFESIONALES Y LA FAMILIA ANTE LA DIVERSIDAD FUNCIONAL DESDE LA REVISIÓN TEÓRICA
}

\author{
J. E. L. Prado ${ }^{1, *}$, A. R. Arias Gago ${ }^{1}$, M. A. Melcon Alvarez ${ }^{1}$ \\ ${ }^{1}$ Universidad de León \\ leonl@hotmail.es*
}

Recepción 01/02/2017 - Aprobación 29/09/2017

DOI: $10.15628 /$ holos.2017.5592

\section{RESUMO}

Son escasos los estudios que tratan la relación entre la familia, la discapacidad y los profesionales de la educación y la salud. El presente trabajo tiene como principal objetivo la revisión de los estudios sobre el papel de las familias de las personas con diversidad funcional y su relación con los profesionales implicados. Pretendemos una reflexión sistematizada de la estimación cuantitativa sintética de todos los estudios disponibles, estableciendo un marco teórico sobre la situación de las familias de personas con diversidad funcional y su relación con los profesionales de la salud y la educación. En este estudio se aportan valoraciones personales acerca de las variables anteriormente citadas. Dentro de las conclusiones obtenidas, se evidencia la relevancia de la cooperación conjunta de familiares y profesionales para una educación eficaz, con una implicación más significativa por parte de los padres en escuela.

PALAVRAS-CHAVE: Discapacidad, afrontamiento familiar, diversidad funcional, educación

\section{THE ROLE OF THE PROFESSIONAL AND THE FAMILY BEFORE THE DIVERSITY FUNCTIONAL FROM THE REVIEW THEORETICAL}

\begin{abstract}
There are few studies dealing with the relationship between the family, disability, and health and education professionals. He present work has as main objective it review of them studies on the role of families of people with functional diversity and its relationship with the professionals involved. We want a systematic reflection of the synthetic quantitative estimate of all of the available studies, establishing a theoretical framework on the situation of the families of people with functional
\end{abstract}

diversity and its relationship with health and education professionals. In this study provides personal estimations about the above mentioned variables. Within them conclusions obtained, is evidence the relevance of the cooperation joint of family and professional for an education effective, with an involvement more significant from the parents in the school. 


\section{APRESENTAÇÃO}

El planteamiento de la familia con uno de sus miembros con discapacidad ha sufrido diversos cambios a lo largo del tiempo. En el pasado era visto como algo negativo, peyorativo a nivel social, tratando de ocultar la discapacidad al resto de la sociedad, por vinculación religiosa o cultural. Hoy en día, estos tiempos oscuros han quedado atrás, ya que, en 1974 la UNESCO (Van Steenlandt, 1991; Ramírez et al., 2012), desarrolla la Educación para la Comprensión, la Cooperación y la Paz Internacionales y la Educación relativa a los Derechos Humanos y las Libertades Fundamentales. Así mismo, el concepto de discapacidad (Amate y Vásquez, 2006) queda enmarcado dentro del enfoque de la rehabilitación, cuyo antecedente lo encontramos en Rusk (1964) que, tras la segunda guerra mundial, inaugura un centro para convalecientes, originándose un movimiento panamericano basado en el tratamiento médico.

Posteriormente, en los trabajos de Amate et al, (1979); Wood (1980 cit. en Amate y Vásquez, 2006) se fijarían las características de los distintos tipos de discapacidad y su clasificación a nivel internacional, con el sobrenombre de Clasificación Internacional de Deficiencias, Discapacidades y Minusvalías (CIDDM) dentro de la Organización Mundial de la Salud (OMS). No obstante, no será hasta 2001 cuando se aporte la nueva publicación de la Clasificación Internacional del Funcionamiento, de la Discapacidad y de la Salud (CIF) (véase Jiménez, González y Martín, 2002).

\section{METODOLOGIA}

El presente artículo tiene como principal objetivo la revisión de los estudios sobre el papel de las familias de las personas con diversidad funcional y su relación con los profesionales implicados. Pretendemos una reflexión sistematizada de la estimación cuantitativa sintética de todos los estudios disponibles, estableciendo un marco teórico sobre la situación de las familias de personas con diversidad funcional y su relación con los profesionales de la salud y la educación, así como los aspectos conflictivos entre los progenitores y los profesionales, asimismo así como la interacción entre sus competencias en la educación del hijo con diversidad funcional. En este estudio se aportan valoraciones personales acerca de las variables anteriormente citadas.

\subsection{Conflicto en la conexión paternal}

Dentro del campo de la conexión paternal, podemos constatar que un sin fin de sentimientos polivalentes e intensos tienen lugar desde el momento en que se tiene la noticia de la discapacidad del hijo hasta el afrontamiento y la asimilación (Mayo, 2010). La investigación de Ferrel (1990), muestra los sentimientos originados en los progenitores al tener constancia de la diversidad funcional de alguno de sus hijos, y los diversos sentimientos que experimentan a lo largo de la vida, siendo variable la intensidad o secuenciación de los mismos. Estos sentimientos varían, según algunos autores, en grado e intensidad. Pero poseen en común diversas fases progresivas. En el caso de Ferrel (1990), se caracterizan por: shock, negación, cólera, ansiedad y tristeza; equilibrio o adaptación y reorientación. Otras características que se pueden destacar son: el desconcierto, la inseguridad, la desilusión, la culpa, el dolor, el miedo, la rabia, el rechazo social, entre otros (MEC, 2012). 
Estos primeros sentimientos con vinculación negativa pueden combinarse con otros con tendencias positivas, al igual que sucede con las emociones (Peniche, 2015). El ser humano como ser emocional y social, puede desarrollar emociones contrapuestas.

Podemos confirmar, tal y como indica Balluerka, et al., (2013, p. 112) en su investigación expuesta en el I Congreso Internacional de Ciencias de la Educación y Desarrollo en España: concluyéndose que, cuando las emociones en las aulas se gestionan debidamente, se logra que el rendimiento académico sea mayor.

Según Balluerka, et al., (2013) y Aritzeta, et al., (2016), para tratar la capacidad de adquirir un mayor rendimiento escolar trabajando el desarrollo de competencias emocionales hay que considerar que no supone que no haya alumnos con malos resultados, pero la nota media de todos los alumnos del aula es más alta, si se trabaja la educación emocional, el alumnado con ambiente emocional positivo en el aula muestran una mayor versatilidad en los procesos de enseñanza-aprendizaje y logran mejores resultados académicos que quienes no se sienten a gusto con el aula y están en aulas de ambiente emocional negativo. En definitiva, la educación emocional facilita el trabajo de procesar información y hace más comprensible lo aprendido.

Además, un estudio destacado en la revista "Nature", de los investigadores Redondo, et al., (2014), revela que el circuito cerebral en el control de los recuerdos se vincula con las emociones tanto positivas como negativas. Los resultados demostraron que un circuito neuronal que conecta el hipocampo y la amígdala (LeDoux, 1999; Goleman, 2013) juega un papel crítico en la asociación de la emoción con un recuerdo. Además, descubrieron que es posible manipular la vinculación emocional de recuerdos concretos por medio de la optogenética en la manipulación neural.

El poder de las emociones juega especial relevancia en la generación de los recuerdos y en la percepción de los mismos, teniendo un interés especial en las personas con discapacidad visual que frecuentemente usan la memoria para la interacción con el entorno. En la ejecución del recuerdo para una persona invidente la asociación con los sonidos y los aromas es significativa al igual que las emociones vinculadas con los sucesos, destacando el hecho de que las emociones negativas son más impactantes y duraderas en la memoria. En el sentido del olfato, tras pasar por el bulbo olfativo, se activa el área corticomedial de las amígdalas, de tal modo que las amígdalas detectan toda experiencia sensorial, jugando especial interés estos aspectos para la educación y la adquisición de conocimiento. Es necesario usar estos recursos en el aula para dotar al alumno de una mejora significativa en el aprendizaje. Solemos vincular distintas emociones y actitudes con lo que nos rodea, siendo los sentidos lo que nos vincula con el mundo. En este sentido, cabe destacar la obra de Boyd (2009) por la asociación de emociones vinculadas con los aromas, y la personalidad de las personas. La educación emocional tendría que tener presente con estos aspectos, para todo el alumnado y con especial interés en el alumnado invidente, donde el sonido y el olfato, son una herramienta más de contacto con el mundo que nos rodea, junto al tacto. Disponer de una educación en la que se valoren tales aspectos es de vital importancia para educar al alumnado en la mejora en su desarrollo personal y bienestar emocional.

Dicho esto, podemos evidenciar que se desarrollan complejas y estrechas interacciones entre los recuerdos y las emociones, como son: el amor, la ternura, el orgullo, alegría, satisfacción con lo logros obtenidos, sentir un desafío continuo ante barreras o limitaciones 
socio-culturales. El poder expresar todas estas preocupaciones y pasiones, es parte esencial en la comunicación entre los padres y los profesionales en el campo de la educación y la psicología.

Según palabras de Winnicott $(1979$, p. 4) "los niños pueden aprender a vivir con una deficiencia, pero no pueden vivir sin la convicción de que sus padres los consideran dignos de amor...sólo así pueden creer que los otros lo amarán en el futuro". Los progenitores requieren de "la capacidad suficiente para realizar el apego y establecer los primeros vínculos...y para promocionar y apoyar al niño en el, a veces, doloroso pero ineludible, camino hacia la independencia" (Lucerga y Sanz, 2004, p. 17).

La investigadora Núñez (2003) evidencia que una familia con uno de sus miembros con discapacidad posee un elevado riesgo de presentar trastornos a nivel psíquico, no es una afirmación concreta, sino que pueden darse un mayor número de casos de probabilidad de aparición. Los conflictos no se dan en el seno familiar sino en el campo de los recursos, estrategias o capacidades en la adaptación a una nueva situación. Designándose diferentes indicadores de riesgo como son: duelos mal enfocados o afrontados, rencores en la pareja, falta de adecuación espacial, exceso de ilusiones, expectativas y deseos, el nivel socio-económico, falta de vínculo familiar o comunicación, negación y evitación, así como el sujeto y su discapacidad forma el núcleo central familiar y la familia solo gira en torno a la discapacidad o aislando al sujeto de la sociedad (Durán, 2011).

Respecto a la integración de las personas con discapacidad en los distintos campos sociales, en especial la integración laboral y educativa. Son diversas las investigaciones sobre los procesos y resultados de los programas aplicados que no satisfacían los objetivos evidenciados en los trabajos de Blanco (1999); Arnaiz (2002); Verdugo (2004); Naicker y García (1998); García y Fernández, (2005); Frutos, (2011); Torres, et al., (2013) y Almendárez y Cedillo, (2014), reflejaban la necesidad de una nueva terminología para las personas con discapacidad.

En el trabajo de Casado y Egea (2000) se evidencia la necesidad por parte de la UNESCO de una nueva terminología para las personas con discapacidad, al igual que la distinción de la educación inclusiva diferenciándose de la integración o la inserción laboral (Castro, et al., 2009). El concepto de inclusión ha sido aceptado por la UNESCO y por el ámbito internacional, sin embargo, la palabra de discapacidad como tal, por su sentido peyorativo se ha visto modificada su terminología a favor de la "diversidad funcional" (Lozano, et al., 2015, p. 21). Este término surgió en el Foro de Vida Independiente, de 2005, con el fin de cubrir la necesidad de una definición más apropiada, justa y adecuada a los tiempos actuales. Se opto por el cambio de antiguas nomenclaturas negativas (minusvalía o discapacidad), aludiendo estás a un desenvolvimiento cotidiano diferente al habitual, a la vez que incorpora el componente discriminatorio que se deriva de este funcionamiento diferente o distinto.

Dentro de los planteamientos Internacionales, junto al plan de la UNESCO, en la Conferencia Mundial sobre Necesidades Educativas Especiales (Samaniego, 2006), un factor destacado es la inclusión en los sistemas académicos, al igual que en las diversas enseñanzas de los diferentes países. Podemos decir que esta delimitación de inclusión está en correlación con las reformas sistemáticas tanto en el colegio como el distrito, así como en la disposición de los gobiernos locales y centrales.

El tema de inserción en el plan general, delimitaba a los sujetos o grupos reducidos, sin remarcar ningún cambio en la mejora de la inclusión de nuevo alumnado, tal como evidencia Díaz 
y Fernández (2005) la distinción entre inclusión e integración se basa en la consideración del individuo como sujeto, en quién reside y se enfoca la "cuestión", en el contexto educativo, intentando satisfacer las demandas de todo el alumnado (Fernández, 2011). No hay que delimitarse únicamente en la inclusión a los factores del ámbito escolar, familiar, o personal. La investigadora Díaz (2005), entiende la inclusión como un término mayor en el campo social, siendo una actitud, un modo de sentir, un modo de valoración, y no en base al tipo de creencias. Actualmente, en ciertos contextos se habla de inclusión en el campo de la innovación, con énfasis en el reconocimiento y atención en la diferenciación y la complejidad dentro de la discapacidad (Díaz y Fernández, 2005; Ruiz y Moreno, 2005; Tejeda y Fernández, 2015).

\subsection{Dicotomía sobre funciones paternales y profesionales}

En los debates que se han desarrollado sobre el papel que desempeñan los profesionales en la calidad de vida de las personas con discapacidad, frente a las figura paterna, es evidente que el papel más importante es el paterno en la educación de su hijo, sea discapacitado o no, siendo insustituibles.

El personal cualificado posee una perspectiva única desde el punto de vista profesional, esto no implica su intervención en todo el espectro de discapacidad. Las acciones interdisciplinares, no sólo entre profesionales sino también con los propios familiares, dan una mayor efectividad en las intervenciones. El profesional tiene que tener presente ciertas precauciones, especialmente si trabaja con cierto aislamiento, accediendo a los recursos posibles y conociendo sus límites. Las culturas institucional y profesional son importantes para conformar el modo en que el profesional define una buena intervención y una buena relación entre la persona y el profesional (Boyt, Gillen, \& Scaffa, 2016).

No obstante, los padres tienen que valorar que la ordenación dentro del sector de la discapacidad pasa por la legislación política, aunque son ellos quienes están legitimados para la defensa de los derechos de la persona con discapacidad. No obstante, los padres no pueden suplantar la labor de los profesionales, especialistas, psicólogos, educadores, auxiliares, personal cualificado de apoyo.

De igual modo, los profesionales (técnicos o políticos) cometerán un error al no valorar la importancia del papel de los padres en la persona con discapacidad, tanto en su educación como en su autonomía. Hay que entender que el profesional lo será en la medida que pueda reconocer los límites que le competen, al igual que su responsabilidad en la función del cargo que desempeña. Aquellos profesionales que inculpan a los padres y que generalizan su culpa, no son profesionales adecuados, ni tampoco lo son los profesionales que designan a la institución como centro exclusivo en la vida de la persona con discapacidad, o que únicamente pueden desempeñar funciones o empleos que los centros les designen.

Cabe citar la innovación del Programa Fotográfico para Personas con Discapacidad Psíquica, englobado dentro del colectivo de FEAPS Madrid, del Centro de Rehabilitación Psicosocial de Carabanchel, donde han podido exponer sus trabajos a finales del 2014 en la Biblioteca Luis Rosales, bajo el título "Tras el objetivo, lo subjetivo", utilizando la cámara como vehículo de comunicación y expresión de pasiones y pensamientos. De tal manera, se lograba romper el aislamiento y los prejuicios sociales sobre el desarrollo profesional utilizando la fotografía como recurso expresivo para manifestar sentimientos personales y lograr una mayor 
comunicación e interacción con las personas. Permitiendo un desarrollo e integración laboral sin estar marcados por sectores manuales o poco cualificados.

Retornando al tema familiar, también caen en un error aquellos padres que se consideran la única voz plausible del sujeto con discapacidad, alegando como defensa que "usted no sabe nada ya que no vive la misma situación". La labor de los profesionales con su larga formación técnica, con su implicación personal y la motivación en su trabajo, tiene mucho que ver en el progreso adecuado de las personas con discapacidad.

En la obra Aprender a vivir, de Marina (2004, p. 8-9) se defiende la necesidad de aunar fuerzas tanto de entes públicos como familiares, en la educación de los hijos. La institución educativa por sí sola no logra dar una cobertura adecuada para la educación. Ha de darse una simbiosis entre institución educativa y núcleo familiar. "Los padres solos no pueden educar a sus hijos, hagan lo que hagan, porque no pueden protegerlos de otras influencias muy poderosas. Los docentes solos no pueden educar a sus alumnos, por la misma razón. La sociedad tampoco puede educar a sus ciudadanos, sin la ayuda de los padres y del sistema educativo. La intervención de padres y maestros es imprescindible, pero todos debemos conocer sus limitaciones y reconocer que, en la tupida red de influencias en que vivimos, todos ejercemos una influencia educativa, buena o mala, por acción o por omisión... Si queremos educar bien a nuestra infancia, es decir, educarla para la felicidad y la dignidad, es imprescindible una movilización educativa de la sociedad civil, que retome el espíritu del viejo proverbio africano: para educar a un niño hace falta la tribu entera" (Peiró, 2005, p. 104).

El papel de la intervención tanto paterna como de los docentes es fundamental en la educación actual. Lograr una educación eficaz depende de la unidad de criterios educativos tanto en el ámbito familiar como en la escuela. Siendo complementarios y continuos, sin rupturas o limitaciones entre ambos núcleos educativos. Es imprescindible la coordinación entre educadores y padres para favorecer la educación de los niños.

Podemos evidenciar, que desde el modelo que aborda la capacitación funcional del ámbito familiar en la calidad de vida, el equipo del Centro Beach de Discapacidad de Kansas (Postón et al., 2003), ha investigado el carácter multidimensional de la calidad de vida en el ámbito familiar, tanto a nivel del sujeto con la discapacidad como a nivel de los familiares que rodean. Estando estas dos tipologías de calidad de vida estrechamente relacionadas, y con influencias notables por causas socioculturales y personales (Schalock y Verdugo, 2002; Samuel, et al., 2012; Hardman, et al., 2013).

Dentro de las pautas de intervención cabe nombrar, "la investigación de las familias con hijos menores de seis años con alguna discapacidad" de Gómez (1999), desarrollado dentro del Equipo de Investigación Sociológica (EDIS), aportando datos en un informe contrastado, distinguiéndose en sus premisas, que la mayoría de los sujetos los encontrábamos en entornos urbanos, tan solo un $11 \%$ estaba en las zonas rurales, encontrándose un mayor número de dificultades por una menor cobertura en servicios. Las demandas de las familias en los primeros años de la infancia de sus hijos se centran en la prevención y el diagnóstico precoz en los niños, la información y formación por parte de los padres, la atención sanitaria, la rehabilitación, así como la certificación de la minusvalía, las guarderías con formación concreta (Pérez, 2008).

Las dificultades halladas por los padres en el ámbito de la educación de sus hijos con discapacidad ya se evidencian en el rechazo al ingreso en algunas guarderías. Esto supone un 
hándicap social más, que los padres se encuentran en los primeros años de la vida de sus hijos, únicamente superando a través de la sensibilización, formación y mejora continua en la especialización por parte de grupos de formación educativa. Cabe exponer el Plan Integral de Apoyo a la Familia (PIAF) donde se engloba la actuación que la familia tiene respecto a la persona con discapacidad. En su estructura recoge desde los servicios de información, formación y orientación, así como el diseño de programas específicos, los servicios de ayuda a los hogares, y las diversas ayudas (Sarto, 2001).

Dentro de la intervención con el entorno de las familias, nos centraremos en la intervención in situ que tiene que ver con los familiares y los padres de la persona con discapacidad.

Dentro de los tipos de intervención en las familias con discapacidad, hay que decir, que los programas son abiertos a mejoras continuas con acciones simultaneas (véase tipos de programas en Fantova, 2000). Hay que decir, que si bien la conceptualización que desempeña el autor en el tipo de intervención es una enumeración breve enmarcada en las predisposiciones y tendencias de los estudios (Boutin y Durning, 1997; Cunningham y Davis, 1988; McConkey, 1985; Kravetz, et al., 1990; Kozloff, 1991; Baker, 1997). Si tienen diversidad en tanto a ser propuestas de valor personal para el autor, puestas en duda en discusiones de trabajos posteriores (Fantova, 2000; Verdugo y Rodríguez, 2009).

Tenemos que entender que, el primer contexto social es el familiar, donde se da seguridad y aprendizaje.

"La familia es el primer contexto de aprendizaje de las reglas sociales y, por tanto, el primer agente socializador de los valores que adquieren sus miembros" (García, Ramírez y Lima, 2001, pp. 204).

Es donde el desarrollo va más allá de lo educativo, entrando en relación diferentes variables, como el aspecto intelectual, social-físico o afectivo, además de las experiencias y vivencias personales. El primer contacto con el entorno fuera del núcleo familiar lo proporciona la propia familia, favoreciendo nuevas relaciones y diversas situaciones (Parada, 2010). El comportamiento, la actitud del sujeto con discapacidad, también estarán en gran medida condicionadas por la relación familiar, siendo el principal modelo a seguir, así como el aprendizaje en la capacidad de resolver conflictos que se afronten en la vida de la persona con discapacidad.

Es por ello, que la familia tiene que favorecer el desarrollo de competencias y habilidades tanto sociales como personales, que permitan la configuración del sujeto y la posterior formación de su personalidad a lo largo del desarrollo de su infancia y adolescencia. Queda patente el gran papel que desempeña el vínculo familiar en el desarrollo del sujeto, aunque no se deba olvidar a los profesionales adecuados que también colaboran para una correcta educación y desarrollo personal (véase, González, et al., 2003).

No podemos generalizar sobre las familias, cada caso es único y particular, no podemos caer en el error de comparar una familia con otra. No obstante, pueden darse ciertas características comunes en el ámbito familiar, pero la función de los profesionales es implicarse de manera individualizada, y sensibilización respecto a la temática de la discapacidad. 
En ciertos casos se dan desadaptaciones, como puede ser el nacimiento de un hijo con discapacidad, cuando los padres tienen que afrontar la situación, bien a través de la aceptación o el rechazo, así como la culpabilidad, y no conseguir las expectativas contempladas (Véase Mayo, 2010). El investigador Caplan (1980, p. 93) lo considera como "un período de desequilibrio psicológico en personas que enfrentan circunstancias peligrosas; esas circunstancias constituyen para el sujeto un problema importante que por el momento no puede evitar ni resolver con los recursos acostumbrados". Afrontar esta "crisis" en cuanto al conocimiento de la discapacidad de un miembro de la familia entraría dentro del grupo de crisis circunstanciales o accidentales, destacando por un carácter inesperado e imprevisto. Este afrontamiento dentro de la familia implica un fortalecimiento familiar, una madurez y crecimiento personal considerables (Mayo, 2010).

Hay unanimidad en el papel fundamental que desempeña la familia con la persona con discapacidad, siendo los padres modelos a imitar por sus hijos, el trato que le den a la discapacidad será un punto de referencia constante en el que las personas con diversidad funcional se fijen. Han sido Arumi (1988), Cantavella (1988), Martínez (1999) y (Mayo, 2010), los que han destacado el mejor modelo de comunicación con los padres, ante las fases de la noticia del hijo con discapacidad, aunque lo hacen desde el ámbito de la investigación (Ammerman, 1997; Gómez, 1999; Pereda, 2012; Pescador, 2013; Guañarita, 2014) destacándose el papel fundamental de la educación (Sarto, 2001).

"En las familias, además de luchar por el desarrollo de todas la capacidades del hijo con discapacidad, debemos continuar con nuestra independencia habitual. La familia no está discapacitada" (Marisa Pérez, Tejada, madre de personas con discapacidad, cit. Sarto, 2001, pp. 1).

En las primeras etapas de intervención tras conocer la noticia, juega especial relevancia la intervención de los profesionales, cuya labor se basa en "acompañar y apoyar a los padres en estas difíciles etapas, mientras que se van dando sugerencias y orientaciones encaminadas a favorecer el desarrollo del niño y facilitar la vinculación con él" (Lucerga y Sanz, 2004, p. 13; Gastón, 2010).

"El profesional debe aceptar todo lo que la familia transmita para facilitar a los padres que expresen lo que sienten y proporcionarles seguridad. La ansiedad contribuye a la creación de sentimientos negativos que los padres pueden albergar y que les pueden hacer sentir culpables" (García y Leonhardt, 2008, pp. 31).

Uno de los objetivos de los profesionales ha de ser reducir el tiempo de la adaptación a la circunstancia, para que la familia pueda ser la principal educadora en unos años tan cruciales como la primera infancia de la persona con discapacidad. Es un acierto que algunos programas cuyo origen estaban pensado para gestionarse en centros, se pasasen al entorno más cercano del niño, el hogar, donde se siente más cómodo. Siendo la familia un pilar esencial del programa implementado por los profesionales a diferencia de los programas del pasado que únicamente se desarrollaban por los propios profesionales sin la vital acción familiar (Troncoso, 1994; Pérez, 2008).

Cabe exponer el Libro Blanco de la Atención Temprana (2000), como elemento de referencia de las asociaciones, profesionales, familias y administraciones, cuyo principal objetivo fue resolver las carencias en los recursos empleados y la organización en la gestión de los 
mismos, estando bajo el respaldo del Real Patronato de Prevención y de Atención a Personas con Minusvalía, así mismo, ha sido utilizado por parte de la confederación Española, FEAPS y la Fundación ONCE (Pérez, 2008).

Cabe destacar unas líneas del Libro Blanco de la Atención Temprana (2000), que dicen lo siguiente:

“El conjunto de intervenciones, dirigidas a la población infantil de 0-6 años, a la familia y al entorno, que tienen por objetivo dar respuesta lo más pronto posible a las necesidades transitorias o permanentes que presentan los niños con trastornos en su desarrollo o que tiene el riesgo de padecerlos. Estas intervenciones, que deben considerar la globalidad del niño, han de ser planificadas por un equipo de profesionales de orientación interdisciplinar o transdisciplinar" (Rodríguez y Zehag, 2009, pp. 37).

Podemos decir, por tanto, que es un momento crucial cuando la familia se entera de la discapacidad de su hijo, porque es cuando surgen los riesgos de ruptura familiar con propensión a ciertas pautas de conducta, donde los efectos psicológicos y sociales son considerables, al igual que la auto-inculpación ante tal situación. Para Caplan (1980) es en este momento para la familia cuando se da "una estación de paso en un sendero que se aleja o se dirige hacia el trastorno" (Núñez, 2003, p. 134).

Respecto al campo clínico, en lo referente a la intervención de los pediatras, psicólogos, y diversos profesionales en el ámbito familiar, podemos destacar varias causas comunes y frecuentes en las familias con discapacidad (Núñéz, 2003): 1) Problemáticas del niño: problemas de conducta (conductas airadas, impulsivas, agresivas, carencia de interés, aislamiento, apatía, inhibición), limitación en el progreso de un tratamiento eficaz o en el campo educativo. 2) Problemática de los hermanos: sintomatología psicosomática, sobre la adaptación, conductas agresivas, exceso de involucramiento con el discapacitado, problemas escolares. 3) Dificultades de los padres: dudas, inseguridad, falta de confianza en la actividad como padres frente a las condiciones del hijo con discapacidad, desorientación, sentimientos de estrés, e impotencia; elevada sobre-exigencia del hijo; limitaciones en la toma de decisiones; ausencia de colaboración familiar o rechazo, ingreso del hijo en un centro especializado (Mayo, 2010).

\section{CONCLUSÃO}

La familia es el principal núcleo educativo, jugando un papel destacado en la educación del hijo, sea o no discapacitado. No obstante, ha de verse complementada con el apoyo y asesoramiento de los profesionales, para una efectiva educación. Por tanto, es esencial recordar que los padres no pueden suplantar la labor de los profesionales y viceversa, y ha de verse como una tarea conjunta, en cooperación, y no de debate entre competencias o disputa en la inculpación de determinados elementos.

Lograr una educación eficaz ha de ser el objetivo de padres y profesionales, y para ello ha de darse una cooperación, aunando criterios educativos desempeñados tanto en el ámbito del hogar como en la escuela, requiriéndose de la interrelación de ambos entornos. El trato que los padres generen ante la discapacidad será un modelo a imitar por los hijos, así como el modo en que resuelvan los conflictos que les surjan en la vida. 
La inclusión en los sistemas educativos fue una realidad tras la Conferencia Mundial sobre Necesidades Educativas Especiales (1994), en parámetros internacionales, intentando aunar los diversos parámetros que las legislaciones educativas poseen, fomentada a través de los planes de la UNESCO.

La labor de los profesionales de la salud y la educación y su interacción con las familias, que tienen uno o más de sus miembros con discapacidad, es de vital importancia, sobre todo, en el caso de familias más vulnerables desde los inicios hasta todo el desarrollo posterior. Una intervención oportuna puede prevenir elementos de conflicto psicológico, evitando así posteriores intervenciones psicoterapéuticas.

Una familia puede verse sobrepasada por la noticia de que un futuro miembro pueda tener una discapacidad, dado que trastoca los pensamientos, aspiraciones e ilusiones desarrolladas con anterioridad. No obstante, el afrontamiento y abordar la discapacidad desde la sensibilización y la formación con la ayuda de profesionales es la mejor herramienta para que el desarrollo del nuevo miembro familiar pueda darse satisfactoriamente.

Por lo tanto, en el desarrollo educativo juega un papel importante la familia pero también los profesionales educativos, psicólogos, especialistas en la discapacidad, que ayudan y asesoran a los padres en la formación de los hijos, su formación tiene que ir a la par, del ámbito escolar al ámbito del hogar, y sería ideal que no se dieran diferencias o distinciones en lo que implica el entorno en el campo de la escuela y la educación recibida en casa por sus progenitores.

Es clave trabajar la educación emocional para el desarrollo de las competencias emocionales en el alumnado sea con o sin discapacidad, cuando se gestionan adecuadamente aumenta el rendimiento académico.

En el caso de alumnado con discapacidad visual, el poder de la educación emocional juega un papel destacado, dado que en la generación de recuerdos, la memoria y las emociones poseen una correlación estrecha, pudiéndose vincular experiencias a través de sonidos y aromas que enriquezcan la didáctica y la generación de recuerdos en el aprendizaje del alumnado invidente.

Se concluye que, la participación paternal en la educación de su hijo con discapacidad es fundamental. Los padres han de tomar conciencia de que poder interactuar en las tareas, ejercicios y actividades con sus hijos con discapacidad implica un nivel de acercamiento que difícilmente se logra dar en el hogar. Por ello, las actividades dentro de la escuela son importantes para el desarrollo, aunque se complemente esta participación con la de los educadores y profesionales cualificados. La separación que existe a día de hoy, entre la escuela y el hogar no tiene que constituir una limitación.

Poder fomentar la participación de las familias en la escuela es clave para que el mensaje que recibe el alumno con discapacidad sea unificado, tanto en el ambiente de casa como en el académico. Los padres han de estimular a sus hijos no únicamente en la etapas iniciales, sino a la largo de toda su etapa educativa, y fomentar actividades extracurriculares que fomenten la motivación y el desarrollo del bienestar personal del individuo. 


\section{REFERÊNCIAS}

Almendárez, L. T. S., y Cedillo, I. G. (2014). Evaluación de un programa de intervención para promover prácticas docentes inclusivas. Revista Actualidades Investigativas en Educación, 14(3), 1-25. Recuperado de: http://revista.inie.ucr.ac.cr/index.php/aie/article/view/629/642

Amate, E. A. y Vásquez, A. J. (Eds.). (2006). Discapacidad: lo que todos debemos saber. Conceptos generales sobre discapacidad. Publicación científica y Técnica. (N. 616). Pan American Health Org. Washington, D.C. ISBN: 9275316163

Amate, E. A., Escudero, M., y Greppi, V. (1979). Rehabilitación: su evolución. En VIII Congreso Latinoamericano de Rehabilitación. Guatemala.

Ammerman, R. (1997). Nuevas tendencias en investigación sobre la discapacidad. En II jornadas científicas de investigación sobre personas con discapacidad. Salamanca, Universidad de Salamanca-IMSERSO.

Arnaiz, P. (2002). Hacia una educación eficaz para todos: la educación inclusiva. Educar en el 2000, 15, 15-18.

Aritzeta, A., Balluerka, N., Gorostiaga, A., Alonso, I., Haranburu, M. y Gartzia, L. (2016). Classroom emotional intelligence and its relationship with school performance. European Journal of Education and Psychology, 9(1), 1-8. doi: 10.1016/j.ejeps.2015.11.001

Arumi, J. D. (1988). Cómo comunicar el diagnóstico de ceguera a los padres. VI Jornadas Internacionales "Entender al bebé hoy". Barcelona: Centre Psicopedagògic. Educaciò Deficient Sensorial, Fundaciò Caixa de Pensions.

Baker, B. L. (1997). Entrenamiento a padres. Siglo Cero: Revista Española sobre Discapacidad Intelectual, 28(173), 13-19.

Blanco, R. (1999). Hacia una escuela para todos y con todos. Boletín del Proyecto Principal de Educación en América Latina y el Caribe, 48, 55-72. Recuperado de: http://educacion.tamaulipas.gob.mx/formacion/cursos_2011/BM12/Hacia_una_escuela.pdf

Balluerka, N., Aritzeta, A., Gorostiaga, A., Gartzia, L., \& Soroa, G. (2013). Emotional intelligence and depressed mood in adolescence: A multilevel approach. International Journal of Clinical and Health Psychology, 13(2), 110-117.

Boutin, G., y Durning, P. (1996). Intervenciones socioeducativas en el medio familiar. Madrid: Narcea.

Boyd, J. (2009). "Perfume y Sensibilidad". Barcelona: Ediciones Perfumerías. ISBN: 8437003 415388

Boyt, B., Gillen, G. \& Scaffa, M. (2016). Terapia ocupacional. (12 Ed). En Ellen. S. Cohn (Coord.). Experiencia de la discapacidad desde una perspectiva familiar. (69-79). ISBN: 9786079356866

Casado, D. y Egea, C. (2000). Las estrategias para el cambio pro inclusión de las personas con discapacidad. Instituto Interamericano del Niño. OEA. 1-7. Recuperado de: http://usuarios.discapnet.es/disweb2000/art/estrategias.htm

Castro, E., Barrero, V., Marín, Y., y Martínez, Y. (2009). Núcleos problemáticos para la inclusión escolar de adolescentes en situación de discapacidad. Umbral Científico, (14), 8-26. Universidad Manuela Beltrán. Bogotá Colombia. Recuperado de: http://www.redalyc.org/pdf/304/30415059002.pdf

Castilla, J. L. (2010). La frecuencia de reforzamiento como moduladora de la acción de variables conductuales y farmacológicas en la polidipsia inducida por programa en ratas. Tesis Doctoral. UNED. Madrid. $\quad 347 . \quad$ Recuperado de: http://espacio.uned.es/fez/eserv/tesisuned:Psicologia-Jlcastilla/Documento.pdf 
Caplan, G. (1980). Principios de psiquiatría preventiva. Buenos Aires: Paidós.

Cantavella, F. (1988). La relación pediatra-padres. VI Jornadas Internacionales "Entender al bebé hoy". Barcelona: Centre Pedagogic Educaciò Deficient Sensorial, Fundació Caixa de Pensions.

Cunningham, C., y Hilton, D. (1988). Trabajar con los padres: marcos de colaboración. Siglo Veintiuno. ISBN: 9788432306501

Díaz, O. C. (2005). Equidad, Inclusión y Discapacidad. Bogotá, D.C. Segundo foro distrital de discapacidad.

Díaz, O. C. y Fernández, A. (2005). Problematización sobre las concepciones vigentes en la educación de las personas en situación de discapacidad. Discapacidad e Inclusión Social: Reflexiones desde la Universidad Nacional de Colombia. Universidad Nacional de Colombia. 301-316.

Durán, M. (2011). Familia y discapacidad: Vivencias de madres y padres que tienen un/a hijo/a ciego/ao sordo/a. Universidad central de Venezuela. Caracas. Recuperado de: http://repositoriocdpd.net:8080/handle/123456789/379

Fantova, F. (2000). Trabajando con las familias de las personas con discapacidad. Organismo especializado de la OEA. Instituto Interamericano del Niño. Siglo Cero: Revista Española sobre Discapacidad Intelectual, 31(192), 33-50. Recuperado de: http://www.iin.oea.org/Cursos_a_distancia/Lectura13_disc..UT3.pdf

Fernández, C.A. (2011). Jóvenes con discapacidades: sujetos de reconocimiento. Universidad de Manzinales. (Tesis doctoral). pp. 1-155. Recuperado de: http://coloquiodiscapacidad.com/investigaciones/phocadownload/Inclusioneducativa/tesis\%20final\%20aleida\%20fernandez\%202011.pdf

Ferrel, K.A. (1990). "Trabajo con Padres". Andalucía Educativa. Junta de Andalucía. Recuperado de: http://www.juntadeandalucia.es/averroes/caidv/interedvisual/ftp/trabajo_con_padres.htm

Frutos, A. (2011). La escuela inclusiva en una sociedad pluricultural y la importancia del trabajo colaborativo. Enseñanza \& Teaching, 28(2), 161-179. Universidad de Murcia. ISSN: 0212-5374. Recuperado de: http://revistas.usal.es/index.php/0212-5374/article/view/7898/8365

Goleman, D. (2013). Cerebro y la inteligencia emocional: nuevos descubrimientos. Ediciones B. Barcelona. ISBN: 978-84-9019-434-8

Gastón, E. (2010). El Nuevo Proyecto Oregón. Integración. Revista de discapacidad Visual. 58. pp. 8. ONCE. ISSN 1887-3383 Recuperado de: http://plataforma.cepmarbellacoin.org/moodle/file.php/376/ESTIMULACION_VISUAL_Y_MUL TISENSORIAL/19_-_Analisis-ELENA_NUEVO_PROYECTO_OREGON.doc

García, A., y Fernández, A. (2005). La inclusión para las personas con discapacidad: entre la igualdad y la diferencia. Revista Ciencias de la Salud, 3(2), 235-246. Bogotá. Recuperado de: http://www.scielo.org.co/scielo.php?script=sci_arttext\&pid=S1692-72732005000200013

García, P., y Leonhardt, M. (2008). Construir juntos espacios de esperanza. Orientaciones para el profesional de atención temprana a niños con ceguera o deficiencia visual. Madrid: ONCE.

García, M.D.; Ramírez, G. y Lima, A. (2001). La construcción de valores en la familia. En M. J. Rodrigo y J. Palacios (coords.). Familia y desarrollo humano. Madrid: Alianza.

Gómez, E. (1999). Las familias con niños discapacitados. Minusval, 120. Instituto Nacional de Servicios Sociales. Madrid: Inserso, (120), 10-17.

González, F., Calvo, M. I., y Verdugo, M. Á. (2003). Últimos avances en el ámbito educativo: Actas $\checkmark$ Congreso Internacional de Educación. Salamanca 4 a 7 Febrero. (Coord.) Francisca González Gil, Ma Isabel Calvo Álvarez, Miguel Ángel Verdugo Alonso. Publicación del Inico. Salamanca. 
pp.

71.

Recuperado

de:

http://campus.usal.es/ inico/newsletter/actasintervencion.PDF\#page=37

Guañarita, C. (2014). La familia como un factor de apoyo en la calidad de vida de las personas en situación de discapacidad [Recurso electrónico] (Tesis Doctoral). Recuperado de: http://bibliotecadigital.univalle.edu.co/handle/10893/7172

Grupo de Atención Temprana (2000). Libro Blanco de la Atención Temprana. Ed. Real Patronato de Previsión y Atención a Personas con Minusvalías.

Hardman, M., Drew, C., \& Egan, M. W. (2013). Human exceptionality: School, community, and family. Cengage Learning. Canada. ISBN: 987-1-133-58983-9

Jiménez, M., González, P., y Martín, J. M. (2002). La clasificación internacional del funcionamiento de la discapacidad y de la salud (CIF) 2001. Revista española de salud pública, 76(4), 271-279.

Kravetz, S., Katz, S., \& Katz, S. (1990). A goal directed approach to training parents of children with a developmental disability. The British Journal of Mental Subnormality, 36(70), 17-29.

Kozloff, M. (1991). El entrenamiento a padres: trabajar por aumentar la normalización y prevenir la institucionalización. Siglo Cero, (135), 24-39.

LeDoux, J.E. (1999). El cerebro emocional. Barcelona: Ariel/Planeta

Lozano, J., Cerezo, M. C., y Alcaraz, S. (2015). Plan de atención a la diversidad. Educatio Siglo XXI, Madrid, Alianza, 33(1), 343-346.

Lucerga, R. y Sanz, M. J. (2004). Puentes invisibles. Madrid: ONCE, colección Manuales.

Marina, J. A. (2004). Aprender a vivir. (1ạ Ed.). Barcelona: Ariel. ISBN: 978-84-34444-652

Martínez, R. (1999). La intervención con la familia ante el nacimiento de un niño deficiente visual: el papel de una escuela de padres. Revista de Atención Temprana, 2(1), 30-36.

Mayo, M. E. (2010). La familia ante la discapacidad visual de un hijo: reacciones, afrontamiento y clima. (Tesis Doctoral). USC. pp. 337. Recuperado de http://www.siis.net/documentos/ficha/198963.pdf

Ministerio de Educación, Cultura y Deporte. (2012). Educación Inclusiva: personas con Discapacidad Visual. Formación en Red. MEC. ONCE. Recuperado de: http://www.ite.educacion.es/formacion/materiales/129/cd/unidad_11/mo11_introduccion.ht $\underline{m}$

McCONKEY, R. (1985). Working with parents. Cambridge, Bookiine Books

Naicker, S. M., \& García Pastor, C. (1998). De la retórica a la realidad: la educación inclusiva en Sudáfrica. Revista electrónica de investigación y evaluación educativa, 4(1), 1-15. Recuperado de: http://www.uv.es/RELIEVE/v4n1/RELIEVEv4n1_4.htm

Núñez, B. (2003). La familia con un hijo con discapacidad: sus conflictos vinculares. Arch Argent Pediatr, 101(2), 133-142. Recuperado de: http://www.sap.org.ar/docs/publicaciones/archivosarg/2003/133.pdf

Organización Mundial de la Salud. (2001). Clasificación internacional del funcionamiento, de la discapacidad y de la salud. Ginebra: OMS.

Parada, J. L. (2010). La educación familiar en la familia del pasado, presente y futuro. Educación Siglo XXI, 28(1). Universidad de Murcia. pp. 17-40. Recuperado de: http://hdl.handle.net/10201/27098

Pereda, T. (2012). El impacto de la discapacidad en la familia. Propuestas de intervención sistémica en la relación de ayuda. EVNTF pp. 1-47. Recuperado de: http://www.avntfevntf.com/imagenes/biblioteca/Pereda,\%20T.\%20Trab.\%203\%C2\%BA\%20online\%2011.pdf 
Pérez, L. C. (2008). Los menores con discapacidad en España. Ediciones Cinca. Cermi. CIAP UNED. Madrid. ISBN: 978-84-96889-35-4

Pescador, C. (2013). Implicación de las familias en los procesos educativos de atención a la diversidad. (TFG). Universidad de Valladolid. pp. 1-77. Recuperado de: http://uvadoc.uva.es/handle/10324/4813

Peiró, S. (Coord.). (2005). Nuevos desafíos de la educación. Tomo 1. X Congreso Interuniversitario de la Teoría de la Educación 2004-2005. Alicante: Grupo Editorial Club Universitario. Recuperado de: http://www.editorial-club-universitario.es/pdf/1050.pdf

Peniche, L. M. (2015). Entender las emociones: Una guía para criar hijos sanos y seguros. Grijalbo. (Spanish Edition). Kindle Edition. ISBN: 978-607-31-3311-1

Poston, D., Turnbull, A., Park, J., Mannan, H., Marquis, J., \& Wang, M. (2003). Family quality of life: A qualitative inquiry. Mental retardation, 41(5), 313-328.

Ramírez, M. C., González, I. R., Castro, M. G., y Astorga, B. (2012). Integración escolar de estudiantes con discapacidad motora: Estudio de caso en un establecimiento educativo regular (Tesis Doctoral). Universidad Academia de Humanismo Cristiano. Santiago. pp. 405. Recuperado de: http://bibliotecadigital.academia.cl/bitstream/handle/123456789/2475/tpedif11.pdf?sequenc $\mathrm{e}=1$ \&isAllowed=y

Redondo, R. L., Kim, J., Arons, A. L., Ramírez, S., Liu, X., \& Tonegawa, S. (2014). Bidirectional switch of the valence associated with a hippocampal contextual memory engram. Nature 513. 426-430. Recuperado http://www.nature.com/nature/journal/v513/n7518/full/nature13725.html

Rodríguez, A. J. y Zehag, M. F. (2009). Crecimiento y desarrollo infantil. En Méndez, G. (Eds.) Autonomía personal y salud infantil. (37-38). Madrid: Ediciones Editex. ISBN: 9788497713849

Ruiz, A. S. G., y Moreno, A. F. (2005). La inclusión para las personas con discapacidad: entre la igualdad y la diferencia. Rev. Cienc. Salud/Bogotá (Colombia), 3(2), 235-246.

Rusk, H. A. (1964). Rehabilitation medicine: A textbook on physical medicine and rehabilitation. New York.

Samaniego, P. (2006). Aproximación a la realidad de las personas con discapacidad en latinoamérica. En Pérez, L. (Eds.). Marco referencial. (35-37). Madrid: CERMI. MTAS. ISBN: 84611-1502-3 Recuperado de: http://www.discapnet.es/Castellano/comunidad/websocial/Recursos/Documentos/Tecnica/D ocuments/79216aa9238145598a639e0518e5d808Aproximacionalarealidad.pdf

Samuel, P. S., Rillotta, F., \& Brown, I. (2012). Review: The development of family quality of life concepts and measures. Journal of Intellectual Disability Research, 56(1), 1-16. DOI: 10.1111/j.1365-2788.2011.01486.x

Sarto, M. P. (2001). Familia y discapacidad. III Congreso "La Atención a la Diversidad en el Sistema Educativo". Universidad de Salamanca. Instituto Universitario de Integración en la Comunidad (INICO). Recuperado de: http://campus.usal.es/ inico/actividades/actasuruguay2001/5.pdf

Schalock, R. L., y Verdugo, M. A., (2002). Handbook on Quality of Life for Human Service Practitioners. American Association on Mental Retardation. Washington, DC.

Tejeda, P., y Fernández, A. (2015). Políticas de educación superior en países del Cono Sur. Revista de la Facultad de Medicina, 63 (1), 33-40. ISSN 0120-0011 Recuperado de: http://www.scielo.org.co/pdf/rfmun/v63s1/v63s1a05.pdf

Torres, N., Lissi, M. R., Grau, V., Salinas, M., y Silva, M. (2013). Inclusión educativa: componentes socio-afectivos y el rol de los docentes en su promoción. Revista latinoamericana de educación 
inclusiva, $7(2)$ 159-173. ISSN 0718-5480 Recuperado de: dialnet.unirioja.es/descarga/articulo/4752915.pdf

Troncoso, M. V. (1994). Programas de Estimulación Temprana, en S. Molina García, Ed. Aljibe.

UNESCO (1994). Conferencia Mundial sobre Necesidades Educativas Especiales. En centro de publicaciones secretaria general técnica. Salamanca. Recuperado de: http://unesdoc.unesco.org/images/0011/001107/110753so.pdf

Van Steenlandt, D. (1991). La integración de niños discapacitados a la educación común. Santiago de Chile: UNESCO/OREALC, Editorial Andros.

Verdugo, M. A. (2004). De la segregación a la inclusión escolar. Instituto Universitario de Integración en la Comunidad (INICO). Universidad de Salamanca. España. Recuperado de: http:/www.down21.org/revista/art_prof/A_verdugo

Verdugo, M. A. y Rodríguez, A. (2009). Guía F. Guía de intervención y apoyo a familias de personas con discapacidad. Consejería para la igualdad y el bienestar. Junta de Andalucía. Trama Gestión. (168-169). Recuperado de: http://www.juntadeandalucia.es/export/drupaljda/Guia_de_intervencion_y_apoyo_a_las_fa milias_de_personas_con_discapacidad.pdf

Winnicott, D. W. (1979). Conozca a su niño: psicología de las primeras relaciones entre el niño y su familia (Guías para padres). Barcelona: Paidós. 